\section{Sind Mikrometastasen im Sentinellymphknoten prognostisch relevant?}

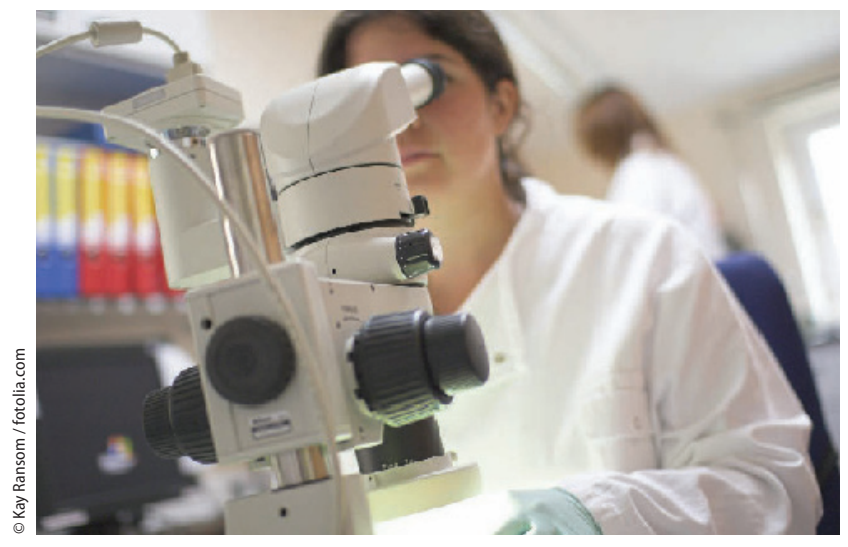

435 Frauen gestorben und 376 hatten ein Tumorrezidiv. Das Vorhandensein von okkulten Metastasen in HE-negativen Sentinellymphknoten hatte keine Auswirkung auf Überleben oder Rezidivrisiko.

Die 5-Jahres-Überlebensrate betrug

Bei Frauen mit einem Mammakarzinom in den Stadien T1 bis T2NOMO hat der Nachweis von Mikrometastasen oder isolierten Tumorzellnestern im Sentinellymphknoten keine Auswirkung auf die Prognose quoad vitam. Auch okkulte Metastasen im Knochenmark sind - wenn andere Risikofaktoren berücksichtigt werden — nicht mit einem kürzeren Überleben assoziiert. Zu diesem Ergebnis kommt eine prospektive Studie mit sechsjähriger Beobachtungszeit.

Für die Studie wurden zwischen 1999 und 2003 an 126 US-amerikanischen Kliniken 5.210 Frauen mit invasivem Brustkrebs im Frühstadium rekrutiert. Bei allen Patientinnen wurden eine brusterhaltende Operation und eine Sentinel-Lymphknotendissektion durchgeführt. Die meisten erhielten außerdem eine adjuvante systemische Therapie. Von 5.119 SentinellymphknotenPräparaten waren 3.904 (76,3\%) gemäß der HE-Färbung tumornegativ. 3326 der tumorfreien Wächterlymphknoten wurden auch immunhistochemisch untersucht. Ob der Wächterlymphknoten befallen ist, spielt scheinbar für die Prognose kaum eine Rolle.

Bei 349 Präparaten (10,5\%) ergab sich ein positiver Befund. 3.413 Patientinnen wurde zusätzlich Knochenmark entnommen. In 104 Biopsaten (3\%) wurden mittels Antikörperfärbung okkulte Metastasen entdeckt. Nach im Median 6,3 Jahren waren
95,1\% — im Vergleich zu 95,7\% bei Frauen, deren Sentinellymphknoten auch immunhistochemisch unauffällig waren. Ob adjuvant systemisch behandelt wurde oder nicht, war ebenfalls ohne Einfluss auf die 5-Jahres-Überlebensrate von Patientinnen mit okkulten Sentinellymphknoten-Metastasen (95,7 vs. 96,3\%).

Frauen mit okkulten Knochenmarks-Metastasen hatten zwar eine höhere Sterblichkeit als Frauen ohne (5-Jahres-Überlebensrate 90,1 vs. 95\%). Aber dieser Unterschied verschwand, wenn andere Risikofaktoren mit in Betracht gezogen wurden. Die mangelnde Signifikanz hängt nach Einschätzung der Autoren möglicherweise mit der begrenzten Zahl von immunhistochemisch positiven Biopsaten zusammen.

\section{Immunhisto kann Hochrisiko-} patientinnen indentifizieren Den Autoren zufolge kann die immunhistochemische Untersuchung des Knochenmarks möglicherweise Hochrisikopatientinnen identifizieren. Für eine routinemäßige immunhistochemische Untersuchung von Knochenmark oder von HE-negativen Sentinellymphknoten bei Frauen mit frühen Brustkrebsstadien (klinisch T1 bis T2N0) gebe es aber keine Berechtigung.

Quelle: Giuliano AE et al (2011) Association of occult metastases in sentinel lymph nodes and bone marrow with survival among women with early-stage invasive in breast cancer. J Am Med Ass. 306: 385-393.
Von diesen Patientinnen wiesen 35\% positive SLN auf.

Aufgrund der unklaren Konsequenzen für die weiteren lokalen und systemischen Therapieentscheidungen kann die Re-SLNB nicht als Standardverfahren beim lokalen Rezidiv nach vorausgegangener SLNB empfohlen werden.

\section{Neoadjuvante Systemtherapie}

Die neoadjuvante Systemtherapie wird zunehmend dazu eingesetzt, die operative Radikalität zu reduzieren. Die Einführung der SLNB hat die Frage aufgeworfen, wie dieses neue Verfahren optimal in der neoadjuvanten Situation eingesetzt werden könnte. Als prätherapeutische Stagingmaßnahme kann die SLNB wichtige Informationen liefern, um die adjuvante Therapie individuell und risikoadaptiert festzulegen. Andererseits wird bei 20-40\% der Patientinnen ein positiver Lymphknotenstatus „sterilisiert“, und hinsichtlich des Nodalstatus findet ein Downstaging statt. Dies setzt jedoch die zuverlässige Prädiktion des Nodalstatus durch den SLN nach vorausgegangener Chemotherapie voraus. Die Daten hinsichtlich der Detektion (Durchführbarkeit der Methode) und der Falsch-negativ-Rate (Sicherheit der Methode) sind in der Literatur jedoch sehr heterogen.

Die deutsche SENTINA-Studie untersucht die Rolle der SLNB in der neoadjuvanten Situation in einem prospektiven Ansatz (• Abb. 1). Über 1500 Patientinnen werden in diese Studie eingeschlossen. Die Studie wird wichtige Informationen über den optimalen Einsatz der SLNB in einem neoadjuvanten Behandlungskonzept liefern.

\section{Klinische Konsequenzen bei positivem Sentinellymphknoten}

Nachdem die ALN als primäre Stagingmaßnahme komplett von der SLNB verdrängt wurde, stellt sich immer mehr die Frage, ob bei allen Frauen, die einen positiven SLN aufweisen, zwingend eine axilläre Dissektion erforderlich ist. Immer- 